CLINICAL STUDY

\title{
Predicting the risk of multiple endocrine neoplasia type 1 for patients with commonly occurring endocrine tumors
}

\author{
Joanne M de Laat, Emma Tham ${ }^{1}$, Carolina R C Pieterman, Menno R Vriens ${ }^{2}$, Johannes A N Dorresteijn, \\ Michiel L Bots ${ }^{3}$, Magnus Nordenskjöld ${ }^{1}$, Rob B van der Luijt ${ }^{4}$ and Gerlof D Valk \\ Department of Internal Medicine, L00.408, University Medical Center Utrecht, PO Box 85500, 3508 GA Utrecht, The Netherlands, ${ }^{1}$ Department of \\ Clinical Genetics, Karolinska Universitetssjukhuset Solna, Sweden, ${ }^{2}$ Department of Surgery, ${ }^{3}$ Julius Center for Health Sciences and Primary Care and \\ ${ }^{4}$ Department of Medical Genetics, University Medical Center Utrecht, Utrecht, The Netherlands \\ (Correspondence should be addressed to G D Valk; Email: g.d.valk@umcutrecht.nl)
}

\begin{abstract}
Objective: Endocrine diseases that can be part of the rare inheritable syndrome multiple endocrine neoplasia type 1 (MEN1) commonly occur in the general population. Patients at risk for MEN1, and consequently their families, must be identified to prevent morbidity through periodic screening for the detection and treatment of manifestations in an early stage. The aim of the study was to develop a model for predicting MEN1 in individual patients with sporadically occurring endocrine tumors.

Design: Cross-sectional study.

Methods: In a nationwide study in The Netherlands, patients with sporadically occurring endocrine tumors in whom the referring physician suspected the MEN1 syndrome were identified between 1998 and $2011(n=365)$. Logistic regression analysis with internal validation using bootstrapping and external validation with a cohort from Sweden was used.

Results: A MEN1 mutation was found in $15.9 \%$ of 365 patients. Recurrent primary hyperparathyroidism (pHPT; odds ratio (OR) 162.40); nonrecurrent pHPT (OR 25.78); pancreatic neuroendocrine tumors (pNETs) and duodenal NETs (OR 17.94); pituitary tumor (OR 4.71); NET of stomach, thymus, or bronchus (OR 25.84); positive family history of NET (OR 4.53); and age (OR 0.96) predicted MEN1. The c-statistic of the prediction model was 0.86 (95\% confidence interval $(95 \%$ CI $) 0.81-0.90)$ in the derivation cohort and 0.77 (95\% CI $0.66-0.88)$ in the validation cohort. Conclusion: With the prediction model, the risk of MEN1 can be calculated in patients suspected for MEN1 with sporadically occurring endocrine tumors.
\end{abstract}

European Journal of Endocrinology 167 181-187

\section{Introduction}

Endocrine diseases such as primary hyperparathyroidism (pHPT) and pituitary tumors (PIT) are relatively common in the general population (1). In addition, the incidence and prevalence of neuroendocrine tumors (NETs) are increasing over the last decades (2). With the increased use of laboratory and radiological investigations, which are sometimes applied for screening purposes, endocrine tumors are also more often diagnosed coincidentally.

Endocrine tumors can occur as part of the autosomal dominant inherited multiple endocrine neoplasia type 1 (MEN1) syndrome. MEN1 is characterized by the occurrence of combinations of: i) pHPT; ii) NET of pancreas and duodenum (pNET); iii) NET of stomach, thymus, or bronchus; iv) PIT; and v) adrenal tumors (ADR). The syndrome is caused by an inactivating germline mutation in the MEN1 gene on chromosome $11 q 13$ (3). Although MEN1 is a rare disease with an estimated prevalence of 1-10/100 000 inhabitants worldwide, timely recognition is of utmost importance because patients are at risk for malignant disease associated with a significant decrease in life expectancy $(4,5,6,7,8,9,10)$. There is growing evidence that an early diagnosis by genetic screening and subsequent preventive care leads to improvement of long-term outcome $(11,12)$. An early diagnosis not only applies for the index patient but also certainly for family members who carry the mutation and are often still symptom free.

The currently used consensus guidelines for diagnosis and therapy of MEN1 (2001) therefore recommend screening of all sporadic patients where MEN1 is suspected (13). This accounts for any patient with two or more MEN1-related tumors, pHPT before age 30, recurrent $\mathrm{pHPT}$, gastrinoma or multiple pNETs, or familial HPT. These guidelines were based only on limited data. In addition, some further small studies have already demonstrated high prevalence of MEN1 mutations in patients with sporadic endocrine tumors, suggesting that the current advises are too 
conservative, which might lead to unnecessary morbidity and mortality $(14,15,16,17)$.

A more reliable identification of patients at risk for a MEN1 mutation is needed to enable more cost-effective screening. Therefore, in the present nationwide study, we assessed the results of all Dutch MEN1 mutation analyses from 1998 to 2010 of patients with sporadically occurring endocrine tumors. We aimed to develop and validate a rule to predict the outcome of MEN1 mutation testing in individual patients with apparently sporadically occurring endocrine tumors based on patient characteristics and type of endocrine tumor.

\section{Materials and methods Study design}

All patients in The Netherlands referred for MEN1 mutation testing between January 1998 and December 2010 were identified (Fig. 1). In The Netherlands, MEN1 mutation testing is done mostly according to the current guidelines that advise to test patients who have two or more possibly MEN1-related tumors, multiple parathyroid tumors before the age of 30, recurrent hyperparathyroidism, gastrinoma, or multiple islet cell tumors at any age or familial isolated hyperparathyroidism. For this study, the outcomes of tests of patients were excluded if: i) MEN1 was clinically diagnosed (at least three out of five endocrine MEN1 manifestations); ii) the patient had a family member with MEN1; iii) all clinical information was missing; or iv) MEN1 mutation testing was performed with an erroneous indication (e.g. non-MEN1-related tumors such as pheochromocytoma, medullary thyroid carcinoma, etc.).

Clinical information was collected from the application form for genetic testing and missing data were retrospectively retrieved from the referring physician. Endocrine manifestations diagnosed after the request for genetic testing were not recorded. Data were collected regarding: pHPT; pNET; PIT; NET of stomach, thymus, or bronchus; ADR; and family history of
MEN1-related (neuro-)endocrine tumors irrespective of the grade of the family relation.

The presence of a MEN1 mutation was determined with DNA sequencing since 1998 and with a combination of DNA sequencing and multiplex ligationdependent probe amplification (MLPA) since 2005. MLPA (MRC-Holland, Amsterdam, The Netherlands) is used for detecting large deletions or duplications in the MEN1 gene.

As missing data may lead to loss of statistical power and bias, multiple imputation based on other determinants as well as the outcome was used to replace missing values for family history $(n=63$, $17.3 \%$ ) (18). The missing data were completely missing at random.

Variables The following variables were considered candidate predictors for a MEN1 mutation: patients' age at the moment of DNA testing; recurrent or multigland disease pHPT; nonrecurrent pHPT; pNET; PIT; NET of stomach, lung, or thymus; adrenal hyperplasia or adenomas (ADR); and a family history of endocrine tumors.

Model derivation The prediction model was established by multivariate logistic regression. In this multivariate analysis, a stepwise backward selection procedure was conducted, excluding predictors with a $P$ value $>0.157$ (Akaike's Information Criterion) $(19,20,21)$. Coefficients and crude odds ratio (OR) with 95\% confidence intervals (CIs) of the multivariate model were calculated to estimate the impact of significant predictors. In general, these measures are too high because the model is developed solely using the study sample and will perform less on a different random sample. Therefore, a bootstrapping procedure was performed to adjust the coefficients. The bootstrap resampling resulted in a mean shrinkage factor of 0.89. Performance of the adjusted model was assessed in the original Dutch cohort by assessing predictive discrimination and calibration. Predictive discrimination was evaluated using the c-statistic that represents the area under the
Dutch cohort

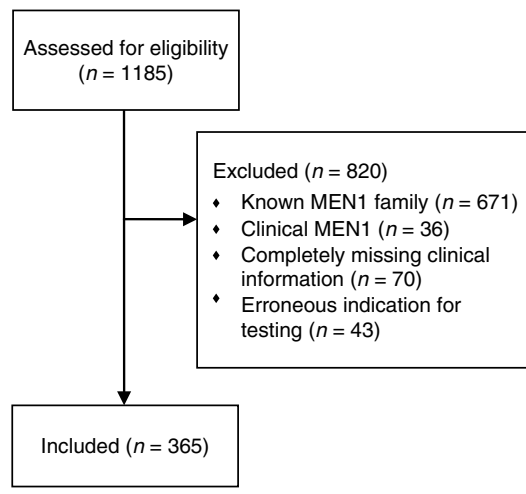

Swedish cohort

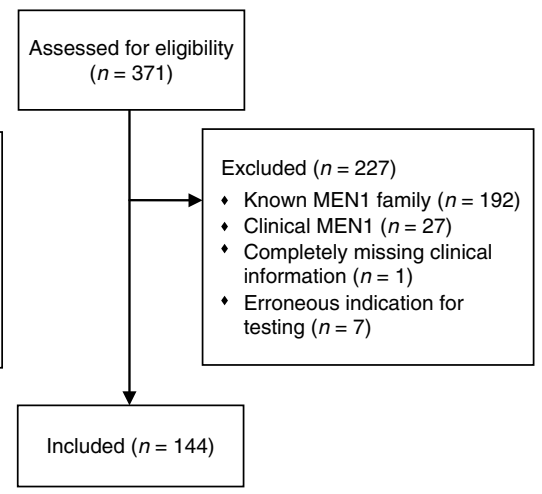

Figure 1 Study flow diagram. 
curve of the receiver operating characteristic curve. To assess goodness of fit, the Hosmer-Lemeshow statistic was computed, which assessed the matching of the predicted and observed MEN1 mutation rate in subgroups of our study population.

\section{External validation}

To evaluate the reliability of the model, it was externally validated in a cohort of patients meeting the same inclusion criteria that was screened between January 1997 and July 2006 at the Department of Clinical Genetics at the Karolinska University Hospital in Sweden (22). The MEN1 gene was also tested by DNA sequencing and MLPA. Data collection and handling of missing data were performed in the same manner as in the Dutch cohort. The predictive discrimination and goodness of fit in the Swedish cohort were presented.

\section{Risk stratification and clinical application}

A nomogram was constructed using the multivariate prognostic model. This nomogram can be used to predict the risk of a MEN1 mutation in individual patients by adding up the scores of each of the risk factors separately. The sum score can then be translated into the individual patients' risk of a MEN1 mutation. Translation into the individual risk is done by drawing a line from the sum score scale to the risk of a MEN1 mutation scale.

Analyses were conducted using SPSS 17.0 (Windows; Chicago, IL, USA) and open source statistical software, $\mathrm{R}$ version 2.9.2 (R Foundation for Statistical Computing, http://www.R-project.org).

The approaches described in this paper did not involve any randomization, experimental intervention, or questionnaire, and the anonymity of patients was not breached. As this article meets the conditions required under Dutch Law (WGBO) for making medical and/or personal data available for statistical or other scientific research, the Medical Ethics Review Committee of the University Medical Center Utrecht concluded that the Medical Research Involving Human Subjects Act (WMO) was not applicable.

\section{Results}

\section{Study population}

Between 1998 and December 2010, a total of 1185 MEN1 mutation analyses were performed in The Netherlands. Of these, the results of the analyses of 36 patients $(3.0 \%)$ were excluded because they had clinical MEN1, 671 (56.6\%) for having a known MEN1 family member, $70(5.9 \%)$ because of completely missing clinical information on the patients, and $43(3.6 \%)$ because of an erroneous indication for ordering an MEN1 mutation analysis. Therefore, the study population consisted of 365 patients (Fig. 1). The baseline characteristics are summarized in Table 1. Mutations in the MEN1 gene were found in $15.9 \%$ of the included patients. There were significantly more females than males in the study population. A total of 131 patients (35.9\%) did not meet the testing criteria of the 2001 consensus guidelines for MEN1 testing. However, among those patients, two MEN1 mutations were found.

\section{Model derivation}

After backward selection in multivariate analysis, the following predictors for a MEN1 mutation were retained in the model: recurrent or multiglandular disease pHPT (OR 162.40); nonrecurrent pHPT (OR 25.78); pNET (OR 17.94); PIT (OR 4.71); NET of stomach, thymus, or bronchus (OR 25.84); a positive family history for MEN1-related (neuro-)endocrine tumors (OR 4.53); and age (OR 0.96; Table 2). ADR was excluded from the model. The c-statistic of the model was 0.86 (95\% CI 0.81-0.90). The Hosmer-Lemeshow indicated a good

Table 1 Baseline characteristics Dutch and Swedish cohort.

\begin{tabular}{|c|c|c|}
\hline Characteristics & Dutch cohort & Swedish cohort \\
\hline Male/female (\%) & $136 / 229(37 / 63)^{a}$ & $56 / 88(39 / 61)^{a}$ \\
\hline Median age in years at mutation analysis (25th, 75th percentile) & $47(35,61)$ & $51(35,61)$ \\
\hline Meeting guidelines MEN1 for DNA testing $(\%)^{\mathrm{b}}$ & 64.1 & 81.3 \\
\hline Mutation detected (\%) & $58(15.9)$ & $19(13.2)$ \\
\hline Recurrent or multiglandular pHPT (\%) & 9.6 & 13.9 \\
\hline Nonrecurrent pHPT (\%) ${ }^{\mathrm{b}}$ & 48.8 & 67.4 \\
\hline Pancreatic NET (pNET; \%) ${ }^{\mathrm{b}}$ & 21.4 & 30.6 \\
\hline NET of bronchus, thymus, or stomach (\%) & 3.8 & 5.6 \\
\hline Adrenal tumor (\%) & 5.2 & 2.1 \\
\hline Family history positive (\%) & 31.5 & 31.3 \\
\hline
\end{tabular}

pHPT, primary hyperparathyroidism; PIT, pituitary tumor.

${ }^{\mathrm{a}}$ Distribution differs significantly within both cohorts $(P<0.05)$.

${ }^{b}$ Percentage differs significantly between the Dutch and the Swedish cohorts $(P<0.029)$. 
Table 2 Prediction model for the presence of a MEN1 mutation. Absolute risk for the presence of a $M E N 1$ mutation $=1 /\left(1+\exp ^{(-b)}\right)$. $b=-4.3100+(1.510 \times$ family history $)+(2.887 \times \mathrm{pNET})+(1.550 \times \mathrm{PIT})+(3.252 \times \mathrm{NET}$ of bronchus, thymus, or stomach $)+(-0.043 \times \mathrm{age})$ $+(5.090 \times$ recurrent or multiglandular $\mathrm{pHPT})+(3.250 \times$ nonrecurrent $\mathrm{pHPT})$.

\begin{tabular}{|c|c|c|c|c|c|c|}
\hline Variable & $\begin{array}{c}\text { Regression } \\
\text { coefficient } \\
\text { (unadjusted) }\end{array}$ & $\begin{array}{l}\text { Odds ratio } \\
\text { (unadjusted) }\end{array}$ & $95 \% \mathrm{Cl}$ & $\begin{array}{c}\text { Regression } \\
\text { coefficient } \\
\text { (adjusted) }\end{array}$ & $\begin{array}{l}\text { Odds ratio } \\
\text { (adjusted) }\end{array}$ & $95 \% \mathrm{Cl}$ \\
\hline Family history & 1.691 & 5.42 & $2.32-12.71$ & 1.510 & 4.53 & $1.93-10.61$ \\
\hline pNET & 3.232 & 25.32 & $8.28-77.47$ & 2.887 & 17.94 & $5.86-54.87$ \\
\hline PIT & 1.736 & 5.67 & $2.23-14.41$ & 1.550 & 4.71 & $1.86-11.97$ \\
\hline NET of bronchus, thymus, or stomach & 3.640 & 38.10 & $6.49-223.85$ & 3.252 & 25.84 & $4.40-151.80$ \\
\hline Age & -0.048 & 0.95 & $0.93-0.98$ & -0.043 & 0.96 & $0.94-0.98$ \\
\hline Recurrent or multiglandular pHPT & 5.698 & 298.27 & $55.77-1595.29$ & 5.090 & 162.40 & $30.36-868.55$ \\
\hline Nonrecurrent pHPT & 3.638 & 38.01 & $11.94-121.06$ & 3.250 & 25.78 & $8.10-82.12$ \\
\hline
\end{tabular}

pNET, pancreatic NET; PIT, pituitary tumor; pHPT, primary hyperparathyroidism.

diagnostic accuracy with a nonsignificant $\chi^{2}$ of 7.3 (P 0.51) using nine groups based on the relatively low MEN1 mutation frequency.

\section{Validation cohort}

Between January 1997 and July 2006, a total of 371 MEN1 mutation analyses were performed at the Department of Clinical Genetics at the Karolinska University Hospital in Sweden. Twenty-seven (7\%) patients were excluded because of clinical MEN1, 192 (52\%) for having a known MEN1 family member, seven (2\%) patients with an erroneous indication for testing, and one because of completely missing clinical information. Subsequently, data from 144 patients were used for external validation of the prediction model (Fig. 1). The baseline characteristics of the Swedish validation cohort differed significantly for the prevalence of pHPT,
PIT, and pNET from the derivation cohort (Table 1). In the Swedish cohort, 27 patients $(18.8 \%)$ were tested for MEN1 and did not meet the current consensus guidelines for MEN1 testing. To compensate for the lower prevalence of MEN1 in the Swedish cohort, the model intercept was adjusted. In this validation cohort, the c-statistic of the Dutch prediction rule was 0.77 (95\% CI 0.66-0.88). The Hosmer-Lemeshow was nonsignificant with $\chi^{2}$ of 14.1 ( $P$ 0.08) using nine groups for goodness of fit based on the relatively low MEN1 frequency (13.2\%).

\section{Model application}

To enable application of the prediction rule, a nomogram was constructed to be used in clinical practice (Fig. 2).

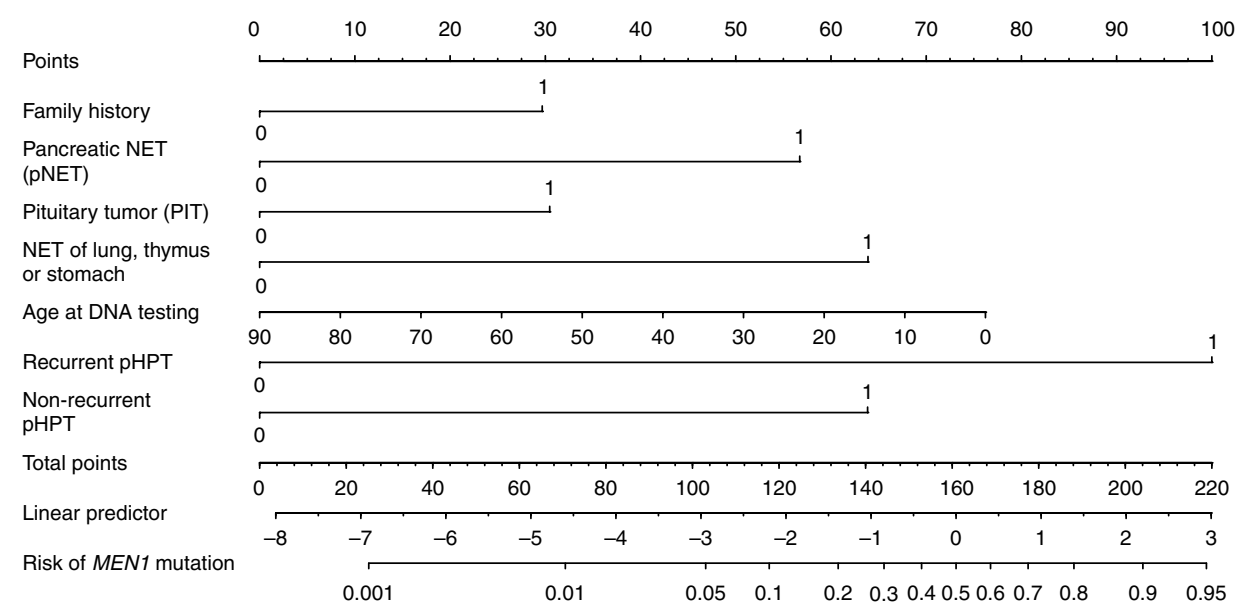

Figure 2 Nomogram. NET, neuroendocrine tumor; pHPT, primary hyperparathyroidism. Example: a 54 -year-old patient (score $=30$ points) with the combination of a negative family history (score $=0$ points), a nonrecurrent and nonmultiglandular pHPT (score $=63$ points), and a pNET ( $n=57$ points) has a sum score of 150 points, corresponding with a linear predictor of -0.50 and a risk of $38 \%$ of having a MEN1 mutation. Example: a 41 -year-old patient (score $=42$ points) with a positive family history (score $=29$ points) and recurrent $\mathrm{pHPT}(\mathrm{score}=$ 100 points) has a sum score of 171 points, corresponding with a linear predictor of 0.50 and a risk of $63 \%$ of having a MEN1 mutation. Example: a 51 -year-old patient (score $=33$ points) with a negative family history (score $=0$ points) of pituitary tumor (score $=31$ points) and a pNET (score $=57$ points) has a sum score of 121 points, corresponding with a linear predictor of -2.0 and a risk of $11 \%$ of having a MEN1 mutation. 


\section{Discussion}

For designing a reliable prediction model and a critical appraisal of the current guidelines of MEN1 mutation analysis, it is important that a large population-based patient cohort can be studied with a high data density and a relatively high number of positive outcomes of mutation analysis. For a rare genetic disorder such as MEN1, this is ideally performed in a truly nationwide study, which is more feasible in a small and very densely populated country such as The Netherlands. As MEN1 mutation analysis in The Netherlands is up to standard and centrally performed at our department of medical genetics, we were able to collect the results of all Dutch DNA tests performed from 1998 to the present day. In addition, before use in clinical practice, a prediction model must be validated in a large independent population-based cohort. For the validation of the prediction model, we therefore used the outcomes of the mutation analysis of another well-described large and completely independent international cohort. In both the Dutch and the Swedish cohorts, patients were tested for a MEN1 mutation because the treating physician suspected MEN1. For various reasons, the current consensus guidelines for MEN1 mutation analysis were not strictly followed in a significant number of patients in both the cohorts. Reasons for screening were a positive family history for endocrine diseases, a relatively young age at which an endocrine tumor occurred, or an atypical course of the endocrine disease, which are not addressed in the 2001 guidelines.

Using all the tested cases, it was possible to design a prediction model that fits in with the current clinical practice. In the Dutch patients who underwent mutation analysis but did not meet the criteria according to the 2001 guideline, two new MEN1 patients (and consequently their pedigrees harboring a MEN1 mutation) were identified. This indicates that the 2001 guidelines for screening of patients with apparently sporadic endocrine tumors that can occur in the course of MEN1 are too restrictive. We were able to identify all MEN1 families in The Netherlands and can therefore be sure that the two presented MEN1 mutations among patients with sporadic endocrine tumors were two new MEN1 index cases.

The previously estimated risk for having a MEN1 mutation in patients with sporadic endocrine tumors ranged from 0 to $67 \%$ depending on the type and number of endocrine tumors; however, those figures were not based upon a truly national database and not validated in an independent population. A more reliable estimation of the overall MEN1 mutation frequency in patients with sporadic endocrine tumors who are tested for MEN1 now turns out to be $\sim 16 \%$. However, it seems more sensible to estimate the risk in individual patients. Given the high number of positive outcomes of the MEN1 mutation analysis, every physician taking care of patients with endocrine diseases should be aware of
MEN1 in a patient with apparently sporadically occurring endocrine tumor(s). With the assumption that the 2001 guidelines for identifying patients at risk for MEN1 are specific but too conservative, we used the total Dutch population that underwent MEN1 mutation analysis to develop a prediction model, which precisely predicts the a priori risk for a MEN1 mutation according to the type of tumor and patient characteristics. Except for adrenal tumors, all endocrine tumors that can occur in the course of MEN1 were predictors for a MEN1 mutation. According to the validation and goodness of fit in the Dutch cohort and Swedish validation cohort, the nomogram can be used to reliably predict the risk for a MEN1 mutation in individual patients in daily clinical practice. We did not consider patients with so-called 'unclassified variants' (UVs) as having a MEN1 mutation. With literature searches and the use of programs for gene prediction, the effect of the changes on the MEN1 gene was estimated for every change in the MEN1 gene. UVs are nonclinically relevant changes of the MEN1 gene that do not lead to the MEN1 syndrome.

There is still ongoing debate on the risk of MEN1 and the different NETs and clinical characteristics. Earlier smaller studies conclude that sporadic cases with the presence of two of the three main related MEN1 tumors and patients with an atypical course of endocrine tumors should be tested. Our findings confirm the contribution of coexisting pHPT and pNET in determining the risk of a MEN1 mutation. In addition, our results show the attribution of each separate endocrine tumor in predicting the presence of a MEN1 mutation. Of these, recurrent or multiglandular pHPT is the strongest predictor (OR 162.40). Cardinal et al. found a mutation rate of $1 / 11(9 \%)$ among patients with sporadic pHPT, which led, for practical reasons, to the conclusion that DNA testing should only be considered for patients below 30 years with pHPT. In our study cohort, 19 MEN1 mutation-positive patients with the solitary endocrine manifestation pHPT were present. All those patients appeared to have one or more additional characteristics such as age below $40(n=6)$ years or a positive family history $(n=11)$ and nine of them had recurrent pHPT. This means that sporadic pHPT is associated with MEN1 especially if such an extra characteristic is present. Our prediction model is applicable for patients with pHPT if they have an additional characteristic such as age of occurrence below 35 years, recurrent or multiglandular pHPT, and/or a family history for endocrine tumors. If a patient does not have such an additional characteristic, the risk of pHPT is overestimated in the model and the outcome cannot be reliably used.

The OR of the predictor age in the model was only 0.96 , which confirms that a younger age is associated with a somewhat higher probability for a MEN1 mutation. In our study, the mean age of the Dutch and Swedish cohort was in line and relatively high at 
48 years. In both the study groups, patients with a family history of MEN1 were excluded, which can explain the age in this study. The addition of the predictor age in our prediction model might lead to a more targeted screening at a younger age, which can subsequently lead to less morbidity in index patients as well as their relatives. Of the endocrine tumors, PIT was the weakest predictor in the model (OR 4.71), and adrenal tumors showed no relation at all.

One of the limitations is the retrospective nature of the study. However, we were able to cross-check the data on the application forms of the referring physicians with the original patient files in $16 \%$ of cases. This concerned the cases that turned out to have a MEN1 mutation or with two out of the following manifestations: pHPT, PIT, and PET. The data reliability was high (concordant in $88.1 \%$ ), indicating that the data that were used for the analysis were accurate. It is not likely that the data accuracy of the other cases is different because the reasons for ordering MEN1 mutation tests were the same for all patients. With regard to the family history, the data density was relatively low, because in daily practice the family history is often not completely taken or registered. Still, our data density with regard to family history was over $80 \%$. A second limitation is that in building the prediction model, we only tested known candidate predictors and were not able to identify other possible candidate predictors like level of hormones or co-occurring skin lesions, because this information was not available. One might also argue about the generalizability of the prediction model. We assume that our prediction model is applicable in other populations within and outside Europe as the model was validated in a true independent cohort and because there are no indications of regional or racial differences in the prevalence of MEN1. Irrespective of the higher prevalence of pHPT in the Swedish cohort and the higher prevalence of PIT in the Dutch cohort, the high outcome of the c-statistic in the Swedish cohort indicates that the model is robust and can be used in other populations, even when there are some differences in the case-mix of patients who are tested.

With our prediction rule and nomogram, the a priori individual risk for a MEN1 mutation in a patient with an apparent sporadically occurring endocrine tumor with reason to suspect MEN1 can be calculated. Our model can be used to make physicians more alert and enable informed decision making for patients. However, it cannot be used for identifying patients who should and should not be tested. Ideally, all MEN1 cases are identified, even those with a low a priori risk. The risk cutoff for ordering a mutation analysis is dependent on the costs on the one hand and the possible consequence for the individual patient on the other hand. Identifying patients with a mutation in the MEN1 gene has important clinical consequences for patients and their families because of the important lifelong surveillance to identify early manifestations of the disease to enable subsequent early interventions. Currently used guidelines for MEN1 testing are appropriate for identifying the patients who are at high risk for MEN1 but showed to be too conservative and might therefore delay timely MEN1 mutation testing. Additional research is needed to evaluate the present prediction model for MEN1 mutation testing. When used in clinical practice, the use of our prediction model can be prospectively validated and the results can be used to improve the current practice and facilitate timely identification of MEN1 patients. Based on our results, the guidelines on MEN1 mutation testing in patients with sporadic endocrine tumors are now open for discussion again.

\section{Declaration of interest}

The authors declare that there is no conflict of interest that could be perceived as prejudicing the impartiality of the research reported.

\section{Funding}

This research did not receive any specific grant from any funding agency in the public, commercial or not-for-profit sector.

\section{References}

1 Golden SH, Robinson KA, Saldanha I, Anton B \& Ladenson PW. Clinical review: prevalence and incidence of endocrine and metabolic disorders in the United States: a comprehensive review. Journal of Clinical Endocrinology and Metabolism 2009 94 1853-1878. (doi:10.1210/jc.2008-2291)

2 Yao JC, Hassan M, Phan A, Dagohoy C, Leary C, Mares JE, Abdalla EK, Fleming JB, Vauthey JN, Rashid A \& Evans DB. One hundred years after "carcinoid": epidemiology of and prognostic factors for neuroendocrine tumors in 35,825 cases in the United States. Journal of Clinical Oncology 200826 3063-3072. (doi:10.1200/JCO.2007.15.4377)

3 Lemos MC \& Thakker RV. Multiple endocrine neoplasia type 1 (MEN1): analysis of 1336 mutations reported in the first decade following identification of the gene. Human Mutation 200829 22-32. (doi:10.1002/humu.20605)

4 Chandrasekharappa SC, Guru SC, Manickam P, Olufemi SE, Collins FS, Emmert-Buck MR, Debelenko LV, Zhuang Z, Lubensky IA, Liotta LA, Crabtree JS, Wang Y, Roe BA, Weisemann J, Boguski MS, Agarwal SK, Kester MB, Kim YS, Heppner C, Dong Q, Spiegel AM, Burns AL \& Marx SJ. Positional cloning of the gene for multiple endocrine neoplasia-type 1. Science 1997276 404-407. (doi:10.1126/science.276.5311.404)

5 Kouvaraki MA, Lee JE, Shapiro SE, Gagel RF, Sherman SI, Sellin RV, Cote GJ \& Evans DB. Genotype-phenotype analysis in multiple endocrine neoplasia type 1. Archives of Surgery 2002 137 641-647. (doi:10.1001/archsurg.137.6.641)

6 Pieterman CR, Vriens MR, Dreijerink KM, van der Luijt RB \& Valk GD. Care for patients with multiple endocrine neoplasia type 1: the current evidence base. Familial Cancer $201110157-171$. (doi:10.1007/s10689-010-9398-6)

7 Dean PG, van Heerden JA, Farley DR, Thompson GB, Grant CS, Harmsen WS \& Ilstrup DM. Are patients with multiple endocrine neoplasia type I prone to premature death? World Journal of Surgery 200024 1437-1441. (doi:10.1007/s002680010237)

8 Doherty GM, Olson JA, Frisella MM, Lairmore TC, Wells SA Jr \& Norton JA. Lethality of multiple endocrine neoplasia type I. World Journal of Surgery 199822 581-586. (doi:10.1007/ s002689900438) 
9 Geerdink EA, van der Luijt RB \& Lips CJ. Do patients with multiple endocrine neoplasia syndrome type 1 benefit from periodical screening? European Journal of Endocrinology 2003149 577-582. (doi:10.1530/eje.0.1490577)

10 Goudet P, Murat A, Binquet C, Cardot-Bauters C, Costa A, Ruszniewski P, Niccoli P, Menegaux F, Chabrier G, BorsonChazot F, Tabarin A, Bouchard P, Delemer B, Beckers A \& Bonithon-Kopp C. Risk factors and causes of death in MEN1 disease. A GTE (Groupe d'Etude des Tumeurs Endocrines) cohort study among 758 patients. World Journal of Surgery $20103 \mathbf{4}$ 249-255. (doi:10.1007/s00268-009-0290-1)

11 Lourenco DM Jr, Toledo RA, Coutinho FL, Margarido LC, Siqueira SA, dos Santos MA, Montenegro FL, Machado MC \& Toledo SP. The impact of clinical and genetic screenings on the management of the multiple endocrine neoplasia type 1 . Clinics 200762 465-476. (doi:10.1590/S1807-5932200700 0400014)

12 Pieterman CR, Schreinemakers JM, Koppeschaar HP, Vriens MR, Rinkes IH, Zonnenberg BA, van der Luijt RB \& Valk GD. Multiple endocrine neoplasia type 1 (MEN1): its manifestations and effect of genetic screening on clinical outcome. Clinical Endocrinology 200970 575-581. (doi:10.1111/j.1365-2265.2008.03324.x)

13 Brandi ML, Gagel RF, Angeli A, Bilezikian JP, Beck-Peccoz P, Bordi C, Conte-Devolx B, Falchetti A, Gheri RG, Libroia A, Lips CJ, Lombardi G, Mannelli M, Pacini F, Ponder BA, Raue F, Skogseid B, Tamburrano G, Thakker RV, Thompson NW, Tomassetti P, Tonelli F, Wells SA Jr \& Marx SJ. Guidelines for diagnosis and therapy of MEN type 1 and type 2. Journal of Clinical Endocrinology and Metabolism $2001 \mathbf{8 6}$ 5658-5671. (doi:10.1210/jc.86.12. 5658)

14 Klein RD, Salih S, Bessoni J \& Bale AE. Clinical testing for multiple endocrine neoplasia type 1 in a DNA diagnostic laboratory. Genetics in Medicine 20057 131-138. (doi:10.1097/01.GIM. 0000153663.62300.F8)

15 Cardinal JW, Bergman L, Hayward N, Sweet A, Warner J, Marks L, Learoyd D, Dwight T, Robinson B, Epstein M, Smith M, Teh BT,
Cameron DP \& Prins JB. A report of a national mutation testing service for the MEN1 gene: clinical presentations and implications for mutation testing. Journal of Medical Genetics 200542 69-74. (doi:10.1136/jmg.2003.017319)

16 Ellard S, Hattersley AT, Brewer CM \& Vaidya B. Detection of an MEN1 gene mutation depends on clinical features and supports current referral criteria for diagnostic molecular genetic testing. Clinical Endocrinology 200562 169-175. (doi:10.1111/j.13652265.2005.02190.x)

17 Tham E, Grandell U, Lindgren E, Toss G, Skogseid B \& Nordenskjold M. Clinical testing for mutations in the MEN1 gene in Sweden: a report on 200 unrelated cases. Journal of Clinical Endocrinology and Metabolism 200792 3389-3395. (doi:10. 1210/jc.2007-0476)

18 Moons KG, Donders RA, Stijnen T \& Harrell FE Jr. Using the outcome for imputation of missing predictor values was preferred. Journal of Clinical Epidemiology 200659 1092-1101. (doi:10. 1016/j.jclinepi.2006.01.009)

19 Atkinson AC. A note on the generalized information criterion for choice of a model. Biometrika 198067 413-418. (doi:10.1093/ biomet/67.2.413)

20 Moons KG, Royston P, Vergouwe Y, Grobbee DE \& Altman DG. Prognosis and prognostic research: what, why, and how? BMJ 2009338 b375. (doi:10.1136/bmj.b375)

21 Royston P, Moons KG, Altman DG \& Vergouwe Y. Prognosis and prognostic research: developing a prognostic model. BMJ 2009 338 b604. (doi:10.1136/bmj.b604)

22 Altman DG, Vergouwe Y, Royston P \& Moons KG. Prognosis and prognostic research: validating a prognostic model. BMJ 2009 338 b605. (doi:10.1136/bmj.b605)

Received 8 March 2012

Revised version received 8 May 2012

Accepted 11 May 2012 